\title{
The Adiabatic Surface Temperature and the Plate Thermometer
}

\author{
ULF WICKSTRÖM \\ SP Technical Research Institute of Sweden \\ Borås, Sweden \\ and \\ Luleå Technical University \\ Luleå, Sweden
}

\begin{abstract}
The concept of adiabatic surface temperature (AST) opens possibilities to calculate heat transfer to a solid surface based on one temperature instead of two as is needed when heat transfer by both radiation and convection must be considered. The adiabatic surface temperature is defined as the temperature of a surface which cannot absorb or lose heat to the environment, i.e. a perfect insulator. Accordingly, the AST is a weighted mean temperature of the radiation temperature and the gas temperature depending on the heat transfer coefficients. A determining factor for introducing the concept of AST is that it can be measured with an inexpensive and robust method called the plate thermometer (PT) even under harsh fire conditions. Alternative methods for measuring thermal exposure under similar conditions involve water cooled heat flux meters that are in most realistic situations difficult to use and very costly and impractical.
\end{abstract}

This paper presents examples concerning how the concept of AST can be used in practice both in reaction-tofire tests and in large scale scenarios where structures are exposed to high and inhomogeneous temperature conditions.

KEYWORDS: surface temperature, plate thermometer, fire resistance.

\section{INTRODUCTION}

The concept of using the adiabatic surface temperature (AST) to characterize a combined radiative/convective heating environment with a single parameter has recently been introduced in fire safety engineering by the author of this paper [1]. The AST is the temperature of a hypothetical surface with a specified emissivity and convection heat transfer coefficient that is exposed to this mixed heating environment and that is backed by thermal insulation (heat conduction through the insulation is zero). The AST is the highest/ultimate temperature that a surface exposed to the specified thermal environment can reach. Thus the sum of the heat transfer by radiation and convection must balance. It has then by definition reached the adiabatic surface temperature. AST is a weighted mean of the radiation and the gas temperatures as will be shown below. It is a very useful concept in fire safety engineering as it indicates ultimate temperature levels and it combines thermal exposure levels by radiation and convection into one effective temperature instead of two, the radiation temperature and the adjacent gas temperature.

Another important feature is that AST can relatively easily be measured using a so called plate thermometer. The standard plate thermometer (PT) was invented to measure and control temperature in fire resistance furnaces and is now specified in the international standard ISO 834 and European standard EN 1363. Thorough investigations [2] and long discussions preceded the introduction of the PT into these standards as the changeover from traditional ways of measuring furnaces temperatures to using PTs meant changes in the severity of the tests in some furnaces. The purpose of the change was, however, accomplished; the thermal exposure in various furnaces when controlled using measurements from PTs is now more or less independent of the fuel and furnace design.

The use of the AST for calculating heat transfer to fire exposed bodies has been demonstrated with tests where a steel beam was exposed to very inhomogeneous thermal conditions including flames and gases with temperatures varying with several hundred degrees [3]. In this very challenging scenario the steel temperature was accurately predicted based on AST calculations obtained from PT measurements only, thus proving that the AST can be measured using a PT. The AST can also be calculated with CFD models like FDS to obtain thermal boundary conditions for analyses of e.g. fire exposed structures [1,4]. As a matter of fact AST can be obtained as direct output from the FDS code. 
The concept of AST as a means for expressing thermal exposure is not limited to scenarios that are relevant to fire resistance of structures. It can also be used at moderate levels of temperature and thermal exposure, as e.g. in a cone calorimeter (ASTM E 1354/ISO 5660). In Ref. [5] it is shown how AST and the incident radiation were calculated based on PT measurements alone, see also below. The incident radiation measured using a heat flux meter as prescribed in the standard was then compared to the incident radiation calculated based on the PT measurements. The two levels of incident radiation thus obtained were almost identical. It could then, of course, be questioned why one should use a heat flux meter when the same measurement can be obtained with a less expensive and much more robust PT for example when controlling the incident radiation in cone calorimeters as well as in many other testing and experimental scenarios. In addition indirectly measuring incident radiation, the PT yields the AST which the author suggests is a significantly more useful parameter to express the thermal exposure level as it combines the thermal insult by radiation and convection. AST results could more easily be applied to alternative scenarios where both radiation and convection heat transfer have a considerable influence.

An experiment was conducted in the SBI test apparatus (EN 13823) where the thermal exposure to the specimen surface from the ignition source (a propane sand burner) was measured using a standard PT [5]. The temperature thus obtained was used to specify the boundary conditions of the specimen in the form of the AST as a function of time. This combined convection and radiation boundary temperature was then used as input to a finite element model (FEM) by which the temperature development of a non-combustible specimen was successfully predicted. In this paper it is illustrated how the heat fluxes measured and calculated based on PT measurements also match with radiation levels estimated by a simple flame radiation analysis.

\section{BASIC THEORY}

Heat is transferred from hot fire gases and flames to structures by convection and radiation [6,7]. The contributions of these two modes of heat transfer are in principle independent and must be treated separately. The convective heat transfer depends on the temperature difference, between the target surface and the surrounding gas, and the velocity of the gas, near the exposed surface as well as the shape and size of the surface, while the incident heat radiation on a surface originates from surrounding flames and gas as well as other surrounding surfaces.

Thus the total heat flux $\dot{q}_{t o t}^{\prime \prime}$ to a surface is

$$
\dot{q}_{\text {tot }}^{\mathrm{w}}=\dot{q}_{\text {rad }}^{\mathrm{ar}}+\dot{q}_{\text {con }}^{\mathrm{w}}
$$

where $\dot{q}_{\text {rad }}^{\prime \prime}$ is the net radiation heat flux and $\dot{q}_{c o n}^{\prime \prime}$ the heat transfer to the surface by convection.

The net radiation heat flux, $\dot{q}_{\text {rad }}^{\prime}$ depends on the incident radiation, $\dot{q}_{i n c}^{\prime \prime}$, on the surface emissivity/ absorptivity, $\varepsilon$, and on the fourth power of the absolute temperature $T_{s}$ of the targeted surface as

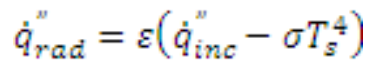

Alternatively, the net radiation heat flux can be written

$$
\bar{q}_{\text {rad }}^{w}=\varepsilon \sigma\left(T_{F}^{4}-T_{g}^{4}\right)
$$

where $T_{r}$ is here termed the black body radiation temperature or just the radiation temperature and can be obtained from the identity

$$
q_{\text {ine }}^{\text {wa }} \equiv \sigma T_{r}^{4}
$$

The heat transferred by convection from adjacent gases to a surface varies considerably depending on the adjacent gas velocities and geometries. However, as radiation heat transfer dominates, especially at higher 
temperatures, and the convective conditions are not decisive for the total heat transfer to fire exposed structures, the heat transfer by convection may be written in a simple form as

$$
\ddot{q}_{\text {con }}^{\mathrm{m}}=h_{\mathrm{e}}\left(T_{g}-T_{s}\right)
$$

The convective heat transfer coefficient $h_{c}$ depends mainly on the flow conditions near the surface.

The total heat transfer to a surface may now be obtained by adding the contributions from radiation and convection. Thus by inserting Eq. 3 and Eq. 5 into Eq. 1 the heat flux to a surface becomes

$$
q_{t o t}^{s g}=\varepsilon_{g} \sigma\left(T_{r}^{4}-T_{g}^{4}\right)+h_{c}\left(T_{g}-T_{g}\right)
$$

Alternatively a radiation heat transfer coefficient can be identified as

$$
h_{r}=\varepsilon \sigma\left(T_{r}^{2}+T_{g}^{2}\right)\left(T_{r}+T_{g}\right)
$$

and Eq. 6 may be written

$$
\ddot{q}_{\text {tot }}^{ \pm}=h_{r}\left(T_{r}-T_{g}\right)
$$

The two boundary temperatures in Eq. 6, $T_{r}$ and $T_{g}$, may now be combined into one effective temperature $T_{A S T}$, the adiabatic surface temperature. This temperature is defined as the temperature of a perfectly insulated surface when exposed to radiation and convection heat transfer [6]. In other words $T_{A S T}$ is defined by the heat balance equation of the surface, i.e.

$$
\varepsilon_{g} \sigma\left(T_{r}^{4}-T_{A S T}^{4}\right)+h_{d}\left(T_{g}-T_{A S T}\right)=0
$$

Note that the value of $T_{A S T}$ is always between $T_{r}$ and $T_{g}$ and that it can be obtained by solving Eq. 9 , an equation of $4^{\text {th }}$ degree.

By combining Eq. 6 and Eq. 9, and assuming that the heat transfer parameters, $\varepsilon_{s}$ and $h_{c}$, of the specimen surface and the PT are equal, the total heat transfer may be written as

$$
q_{t o t}^{s g}=\varepsilon_{g} \sigma\left(T_{A S T}^{4}-T_{g}^{4}\right)+h_{\sigma}\left(T_{A S T}-T_{g}\right)
$$

\section{A Simple Analysis of Heat Transfer from a Flame to a Solid Surface}

In flames and smoke from fires it is the smoke particles and the combustion products that absorb and emit heat radiation. Assuming diffuse gases (not considering wavelength) the overall absorption $\alpha_{f l}$ and emission $\varepsilon_{f l}$ coefficients of a flame or smoke layer depends on the extinction/absorption coefficient $K$ and the depth of the flame or the smoke layer $L$. According to the Kirchhoff's law the absorptivity and the emissivity are equal. Thus

$$
\alpha_{f L}=\varepsilon_{f L}=1-e^{-K L}
$$

The emitted heat from a flame may accordingly be written as

$$
\dot{q}_{e m i, f l}^{\boxplus I}=\left(1-e^{-\mathrm{KL}}\right) T_{f l}^{4}
$$


The emissivity derived above refers to gas layers where the radiation passes straight through the layer with a thickness $L$. In a practical problem of a gas contained between two large parallel plates which emit radiation diffusively the radiant energy transmitted through the gas travels many distances. Thus for a volume between two infinite planes at distance $L$ where the energy is transmitted to not only normal to the surfaces (see e.g. Ref. [6]) a mean beam length $L_{e}$ can be obtained as

$$
L_{e}=1.8 \mathrm{~L}
$$

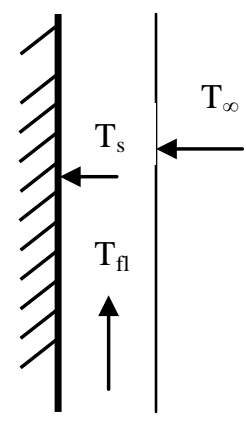

Fig. 1. Illustration of a simple one-dimensional model of heat transfer from a flame to a solid surface.

Then the heat flux by radiation to the surface as shown in Fig. 1 may be written as

$$
\dot{q}_{i n e}^{B D}=\varepsilon_{f l} \sigma T_{f D}^{4}+\left(1-\varepsilon_{f D}\right) \sigma T_{\infty}^{4}
$$

The latter term is the contribution coming from outside the flame and being partly absorbed in the flame. The total heat flux to the surface by radiation and convection now becomes

$$
\ddot{q}_{t o t}^{s b}=\varepsilon_{s} \sigma\left[\varepsilon_{f D} T_{f D}^{4}+\left(1-\varepsilon_{f D}\right) T_{\infty}^{4}-T_{s}^{4}\right]+h\left(T_{\infty}-T_{f D}\right)
$$

Where $\varepsilon_{s}$ is the surface emissivity of the solid. The use of these formulae is demonstrated below.

\section{MEASURING OF INCIDENT HEAT RADIATION WITH A PT IN ISO 5660}

A PT was placed in the specimen holder of a cone calorimeter (ISO 5660) and exposed to radiant heat in horizontal and vertical orientations [5]. The incident flux was set at several levels controlled by heat flux meters according to the standard. Based on the PT temperature, $T_{P T}$, recordings incident flux under steady state conditions was calculated by the simple expression given in Eq. 16.

$$
\ddot{q}_{\text {ine }}^{s g}=\sigma T_{p T}^{4}-\left[h_{c}\left(T_{g}-T_{P T}\right)+K\left(T_{g}-T_{P T}\right)\right] / \varepsilon_{g}
$$

The conduction coefficient $K$ is a proportionality factor found by Häggkvist [11] to be in the order of $K=6 \mathrm{~W} / \mathrm{m}^{2} \cdot \mathrm{K}$ by a thorough thermal finite element analysis of the PT. The surface emissivity $\varepsilon_{s}$ was assumed to between 0.8 and 0.9 . Both these values have been assumed in the calculations reported in the diagrams below. The remaining parameter, $h_{c}$, was calculated according to textbook recommendations (see e.g. Ref. [6]) assuming free convection depending on temperature and size to be between 11.5 and $12.5 \mathrm{~W} / \mathrm{m}^{2} \cdot \mathrm{K}$ for the vertical orientations and between 10.5 and $11.4 \mathrm{~W} / \mathrm{m}^{2} \cdot \mathrm{K}$ for the horizontal orientations. Comparisons between the incident radiant heat flux measured with a Schmidt-Boelter heat flux meter and obtained from PT measurements and insert into Eq. 16 above are shown in Fig. 2 for a horizontally mounted specimen.

It can be concluded from this tentative study that the plate thermometer (PT) may be used for measuring incident heat flux. The accuracy in measuring the incident radiant heat flux with a non-cooled passive device like a plate thermometer is in most practical cases likely to be at least as good or better than measuring with traditional water cooled heat flux meters. Figure 2 indicates that the sensitivity to emissivity is marginal, and 
from Eq. 16 it is evident that the sensitivity could be improved by reducing the factor $K$, i.e. making the insulation of the plate thicker and more efficient, or alternatively if more practical measuring the temperature on the backside face of the insulation to get a better estimate of the conduction losses. Certainly these measurements appear promising although further study is needed to optimize the PT for different applications and to determine the accuracy of such measurements not least in relation to traditional heat flux measurements with water cooled instruments.

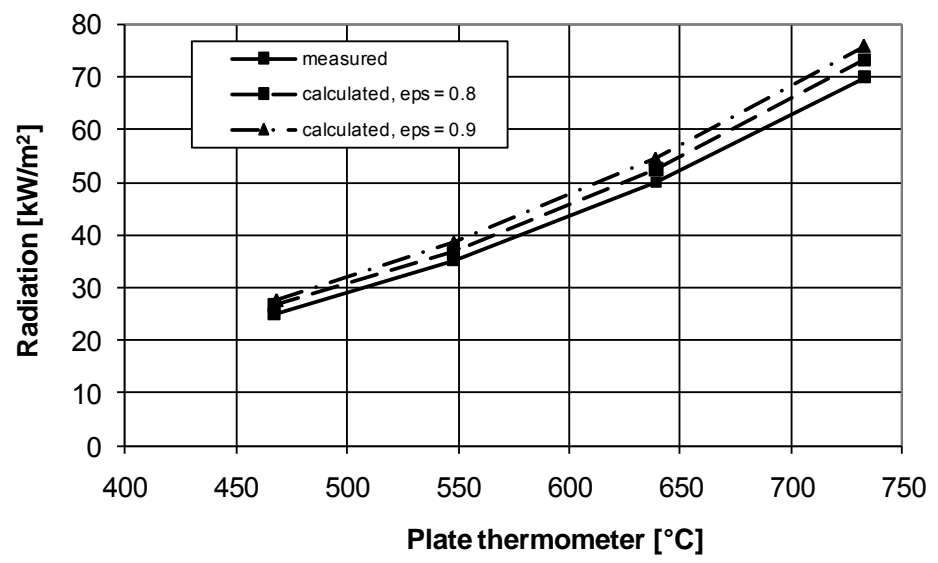

Fig. 2. Comparison between measured and calculated (Eq. 16) incident radiant heat flux, horizontal orientation.

\section{PREDICTION AND ANALYSIS OF THE HEAT TRANSFER IN AN SBI TEST}

A simple experiment was carried out in an SBI test apparatus (EN 13823) with non combustible calcium silicate boards (trade name Promatec H8). On the left hand side of the corner above the propane sand burner, the temperature was measured using a standard Plate Thermometer, see Fig. 3. On the other side of the corner the surface temperature of the board was measured using small thin copper discs. Based on the PT measurements the adiabatic surface temperature (AST) was calculated according to Eq. 9.

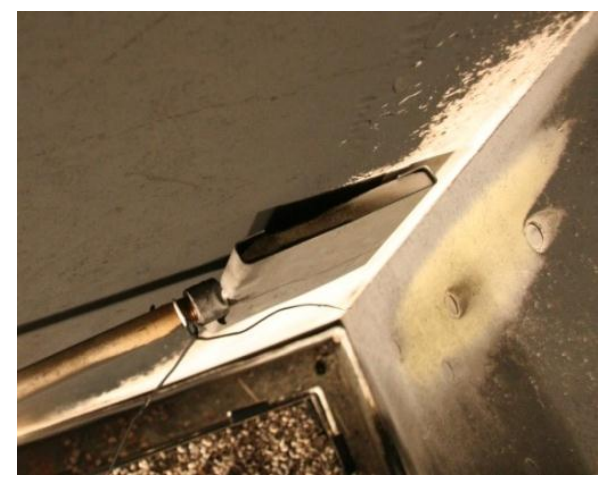

Fig. 3. PT placed to the left of the corner to measure AST and small thin copper disc mounted to the right of the corner to measure surface temperature in the SBI test. The picture was taken after the test.

The term in Eq. 9 containing the coefficient $K$ was neglected as the PT is entirely engulfed in flames and therefore the heat passing between the two surfaces of the PT is negligible. Using this temperature $\left(T_{A S T}\right)$ as the heat transfer boundary condition according to Eq. 10, the calcium silicate board surface temperature $\left(T_{s}\right)$ could be calculated numerically as a function of time using the finite element program TASEF [11], i.e. $T_{s, T A S E F}$. In these calculations constant thermal properties were assumed for the board, i.e. a conductivity of $0.18 \mathrm{~W} / \mathrm{m} \cdot \mathrm{K}$, density of $800 \mathrm{~kg} / \mathrm{m}^{3}$ and specific heat capacity of $840 \mathrm{~J} / \mathrm{kg} \cdot \mathrm{K}$. The calculated surface temperature was then compared with the surface temperature measured using the copper disc at the symmetrically corresponding position at the other side of the corner, $T_{s, \text { meas }}$. The temperatures are shown in Fig. 4. 
Note that the adiabatic surface temperature $T_{A S T}$ is considerably higher than the recorded plate thermometer temperature $T_{P T}$ in the beginning of the test due to the thermal inertia of the PT. Note also that all temperatures converge to approximately the same value. From Fig. 4 it can be seen that, as $T_{A S T} \approx T_{P T}$, the thermal exposure in terms of adiabatic surface temperature at the point in the SBI studied corresponds to an incident radiation level in the cone calorimeter of about $45 \mathrm{~kW} / \mathrm{m}^{2}$.

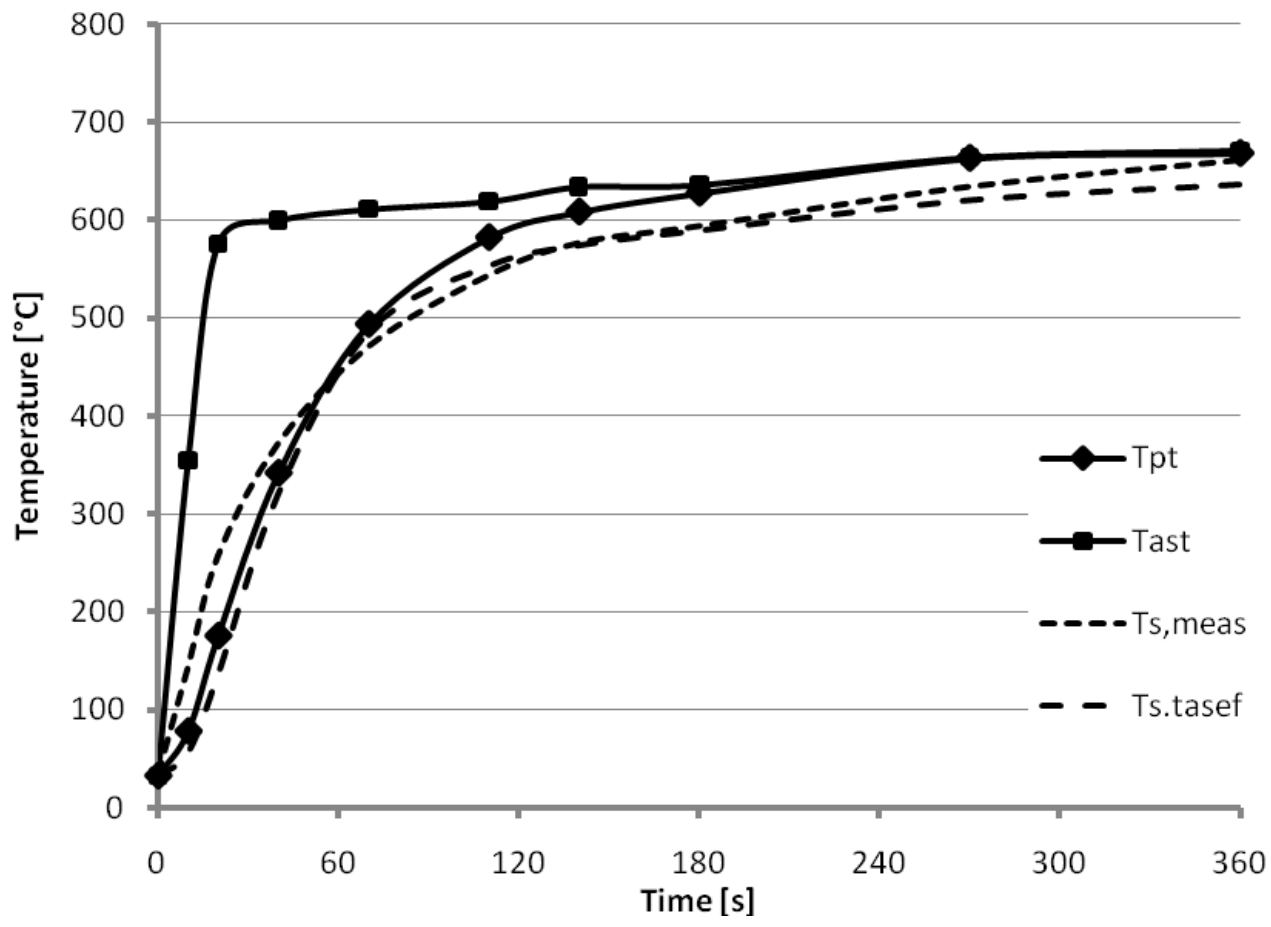

Fig. 4. Comparison between measured PT temperature,$T_{P T}$, calculated AST based on $T_{P T}$, measured specimen surface temperature $T_{s, \text { meas }}$, and calculated specimen surface temperature $T_{s, T A S E F}$.

As a consequence of this type of analysis it can be concluded that a combustible specimen with similar thermal properties as the board tested and an ignition temperature of $400{ }^{\circ} \mathrm{C}$ would ignite at the studied position after about $50 \mathrm{~s}$. A full map of the thermal exposure of the specimen by the ignition source in the SBI test could of course be obtained and compared with AST levels in the cone calorimeter. Thus the thermal exposure of the specimen in the two methods could be directly compared.

The incident radiation can, for comparison, also be estimated from a simple one-dimensional theory using Eq. 11 and Eq. 13. In this case the flame thickness is estimated to be $0.2 \mathrm{~m}$ which yields a mean beam length of $0.36 \mathrm{~m}$ according to Eq. 13. The absorption coefficient for LPG or propane is $0.35 \mathrm{~m}^{-1}$ (see Ref. [8]), and then the flame emissivity can be obtained from Eq. 10 to be approximately 0.13 . Thus, the incident radiation becomes $13.6 \mathrm{~kW} / \mathrm{m}^{2}$ according to Eq. 14 .

However, in the SBI scenario two-dimensional effects must be considered. The two pieces of test specimen can "see" each other. Close to the corner the view factor $\phi$ can be estimated to be almost $50 \%$. The surface temperature is about $650{ }^{\circ} \mathrm{C}$, the surface emissivity $\varepsilon_{s}=0.9$ and thus the incident flux from the opposite side of the corner may be roughly estimated as

$$
q_{s i d e}^{y b} \propto \varepsilon_{s} \sigma T_{s}^{4}=0.5 * 0.9 * 5.67 * 10^{-8}(650+273)^{4}=37000 \mathrm{~W} / \mathrm{m}^{2}
$$

Now the total incident radiation heat flux in the SBI can be roughly estimated by adding the contribution directly from the flame, i.e.

$$
\ddot{q}_{\text {ine }}^{s s}=(37.0+13.6) 10^{3} \approx 50 \mathrm{~kW} / \mathrm{m}^{2}
$$

which is of the same order of magnitude as was measured indirectly with the PT, i.e. $45 \mathrm{~kW} / \mathrm{m}^{2}$. 
Although some of the assumptions are speculative this numerical exercise indicates how the theories based on AST works and how it can be used for calculations and estimates.

\section{PREDICTION OF STEEL TEMPERATURES}

To verify the theory and the concept of using AST and PT in practice three experiments $[3,7]$ were conducted in a small room with dimensions as given in the room-corner standard ISO 9705, i.e. $3.6 \mathrm{~m}$ by $2.4 \mathrm{~m}$ and $2.4 \mathrm{~m}$ high with a door opening $0.8 \mathrm{~m}$ by $2.0 \mathrm{~m}$. In the tests a steel beam was hung $20 \mathrm{~cm}$ below the ceiling along the centre of the room as shown in Fig. 5. A gas burner above the floor, yielding a constant heat output of about $450 \mathrm{~kW}$, was placed in a corner or at the middle of the rear wall. Temperatures were measured in the gas phase with various thermocouples in the gas phase including plate thermometers on the four sides of the beam at three measuring stations along the beam. At the same stations the steel temperature was accurately measured with quick tip thermocouples peened into drilled holes.

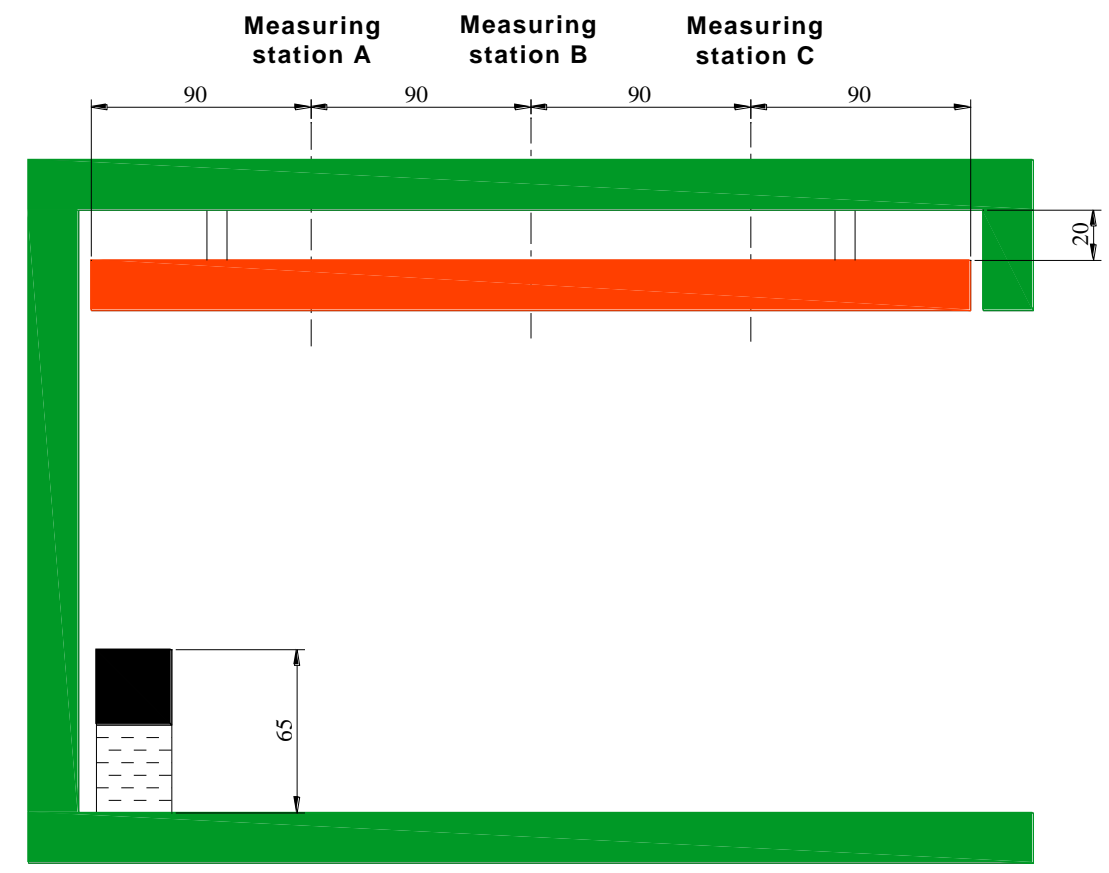

Fig. 5. The ISO 9705 room corner test burn room with a steel beam hanging from the ceiling. At each measuring station there are four PTs and TCs in the gas phase mounted around the beam and several TCs for measuring steel temperatures [3], (measurements in $\mathrm{cm}$ ).

A photo of a plate thermometer with additional quick tip, welded and shielded thermocouples is shown in Fig. 6a. As shown in Fig. 6b, the fire gas field around the steel beam is very heterogeneous and therefore the recorded temperatures at various positions were considerably different. This makes the measurements and mapping of the thermal conditions a complicated challenge. In this case only the plate thermometer recordings were used as input boundary conditions for the theoretical predictions of the steel temperatures presented below. 


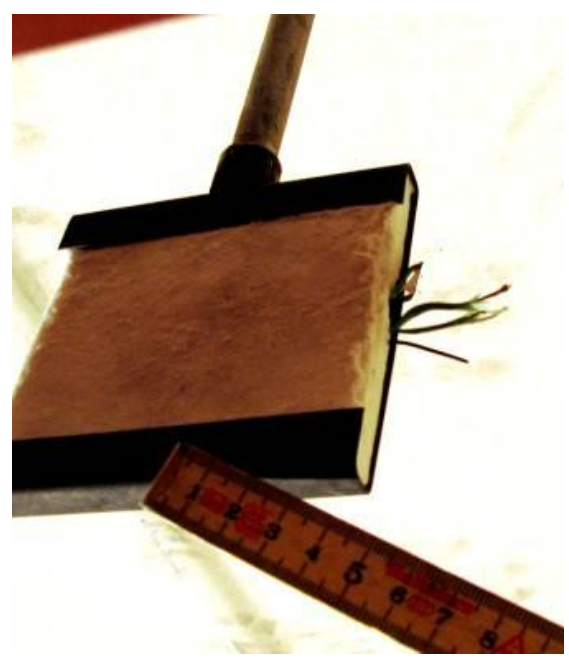

(a)

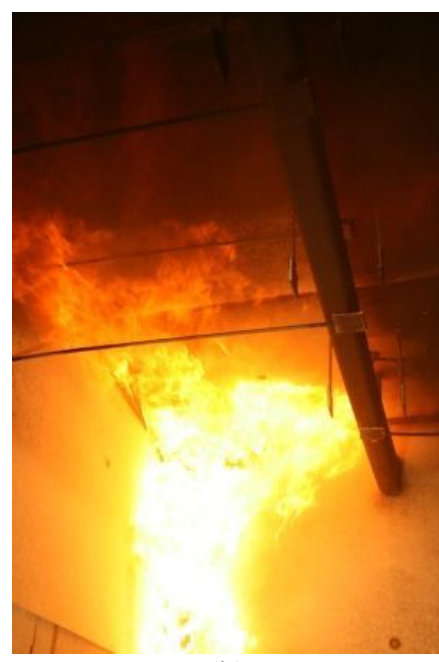

(b)

Fig. 6. Plate thermometer: (a) temperature was measured with PTs and small thermocouples fixed thereto; (b) plate thermometers mounted around the steel beam at three measuring stations, A-B.

Figure 7 shows the temperature measured in the gas phase with fast responding $0.1 \mathrm{~mm}$ welded thermocouples (TC) and plate thermometers (PT) at four positions around the steel beam, Position 1 facing the ceiling, Position 2 facing away from the burner, Position 3 facing the floor and Position 4 facing the burner. All measurements were made at Measurement Station A. For full details see Ref. [3]. Note that at Position 1 the PT is influenced by radiation from the more slowly heated ceiling surface, and therefore the PT temperature is considerably lower than the TC temperatures $\left(\approx 75^{\circ} \mathrm{C}\right)$. Similar behaviour was noted below the beam at Position 3 where the PT is facing the cool floor. On the other hand at Position 4, the burner side of the beam, the PT temperature is almost $100{ }^{\circ} \mathrm{C}$ above the TC temperature. That is due to direct radiation from the burner plume.

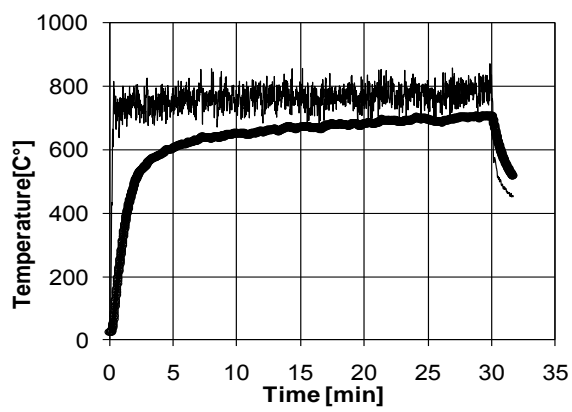

(a)

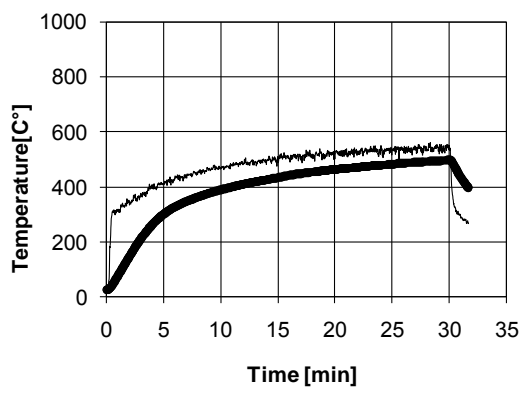

(c)

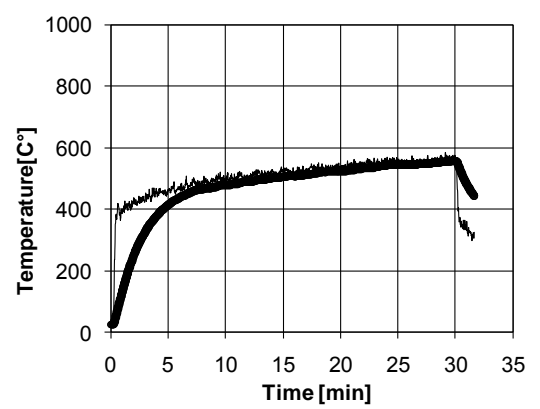

(b)

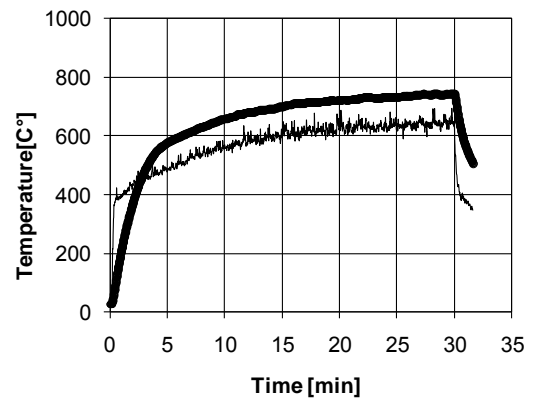

(d)

Fig. 7. Temperature measured in the gas phase at measuring station A with $0.1 \mathrm{~mm}$ welded TCs (thin lines) and PTs (thick lines): (a) Position 1 (facing the ceiling); (b) Position 2 (facing away from the burner); (c)

Position 3 (facing the floor); (d) Position 4 (facing the burner). 


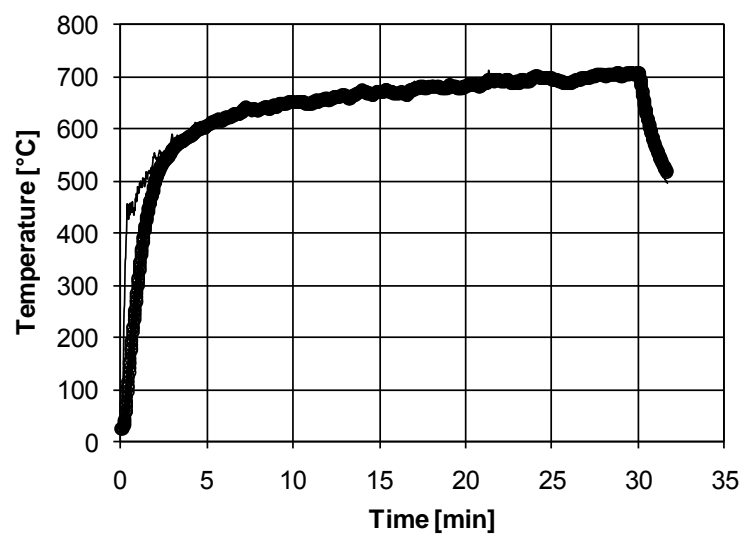

Fig. 8. Comparison between $T_{P T}$ (thick line) and $T_{A S T}$ (thin line) derived from these temperatures for a rapid fire exposure change.

Except when the temperatures changes very rapidly, the plate thermometer measures a temperature very close to the adiabatic surface temperature AST. As outlined in Ref. [7] a more precise value of the AST can be derived from plate thermometer recordings by inverse calculations. That means $T_{A S T}$ is obtained from a transient heat balance model assuming lumped heat capacity of the plate thermometer itself where the boundary condition is as expressed by Eq. 10 and $T_{s}=T_{P T}$ as measured. Figure 8 shows an example of an AST curve derived from a very rapidly rising PT temperature curve where the emissivity and the convection heat transfer coefficient were chosen to be 0.9 and $25 \mathrm{~W} / \mathrm{m}^{2} \cdot \mathrm{K}$, respectively. The choices have only a marginal influence on the calculated AST in the cases studied except for the first few minutes when there is a considerable difference between the PT temperature and the corresponding AST.

Figure 9 shows the four ASTs derived from PT temperatures recorded around the beam. Note that the AST above the beam facing the ceiling (Position 1) rises fastest in the beginning while the AST at the side of the beam facing the burner (Position 4) reaches the highest temperature towards the end of the test. Note that the difference between the hottest AST (Position 4) and the lowest AST under the beam facing the floor (Position 3) is not less than $250^{\circ} \mathrm{C}$.

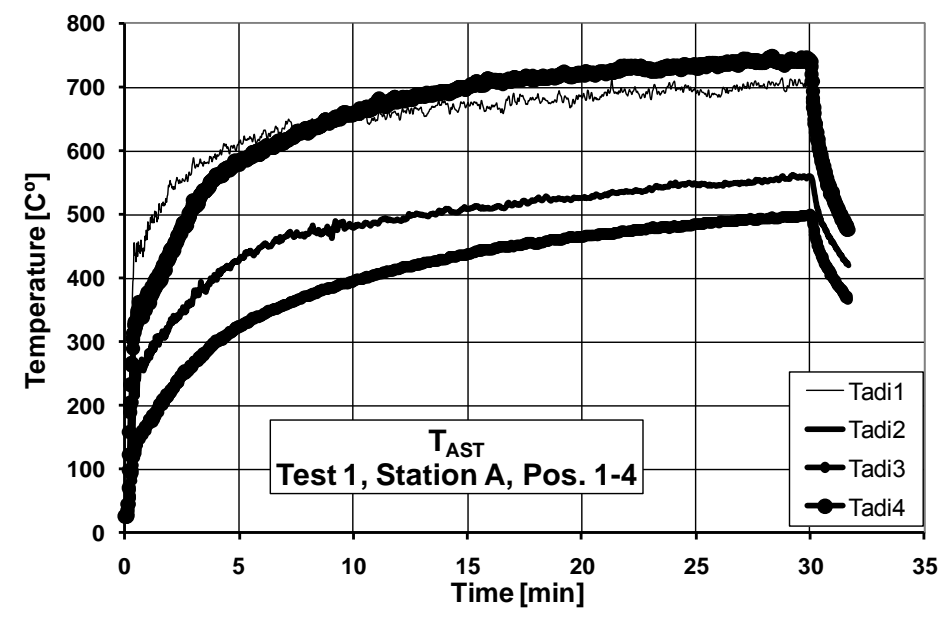

Fig. 9. Collation of AST derived from PT temperatures, Position 1 (thinnest line) through Position 4 (thickest line).

The temperatures of the steel beams were calculated using the 2-D finite element temperature calculation code TASEF [9]. Below the results from the two first tests are reported where the burner is placed in one of the corners of the rear wall. As input to the calculations, the thermal properties of steel were assumed as given in Eurocode 3, EN 1993-1-2 and the ASTs as obtained from PT measurements in the four positions around the steel beams were the only boundary temperatures applied according to Eq. 10. All emissivities and all convection heat transfer coefficients were assumed to be 0.9 and $25 \mathrm{~W} / \mathrm{m}^{2} \cdot \mathrm{K}$, respectively, as recommended in 
Eurocode 1, EN 1991-1-2, for fire exposed structures. This might be seen as a rough assumption, but as seen in Fig. 10 the errors are small.

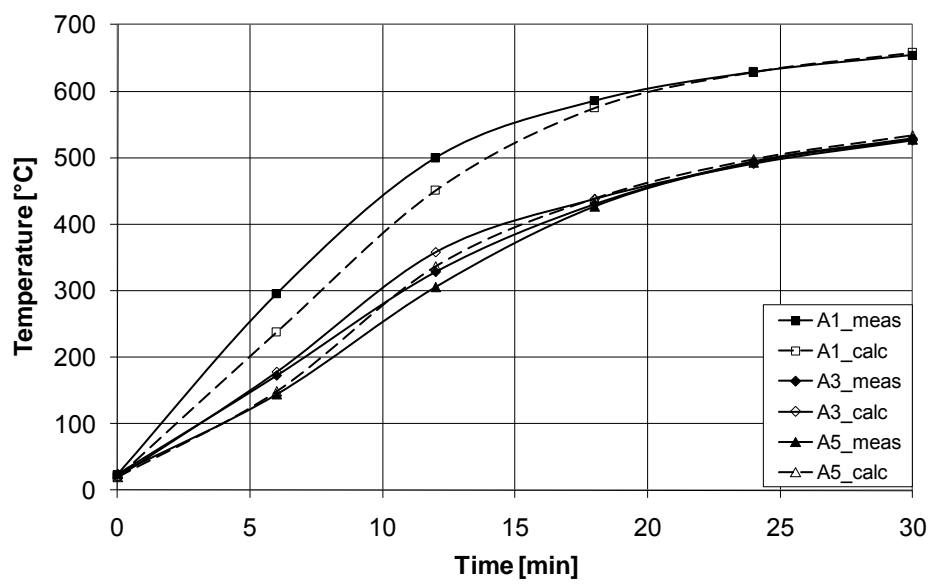

Fig. 10. Measured steel temperature of the square tube section and calculated temperature at the corresponding positions as a function of time [3].

\section{CONCLUSIONS}

The provisional studies reported here show how the concept of adiabatic surface temperature and measurements with plate thermometers can be used for predicting heat transfer to surfaces exposed to radiation and convection. The AST yields a very useful measure of thermal exposure. It can be measured in practice and in addition it can be obtained from fire CFD models like FDS. It is a quantity which straightforwardly can be used for expressing heat transfer by radiation and convection, i.e. the boundary conditions for predicting temperatures in the solid phase. The same surface emissivity and the convection heat transfer coefficient as used in the CFD calculation can then be assumed for the calculations of the heat transfer the solid phase surface. Solid phase temperatures can after that be used, e.g. for estimating times to ignition or loss of strength of structural materials.

Alternative methods for measuring thermal exposure, e.g. measurements of incident radiation or heat flux, are in most realistic cases simply not possible.

This paper has shown that the concept of AST is applicable to fire resistance problems with high thermal exposures as well as to reaction-to-fire problems including incident radiation from flames where e.g. the phenomena like time to ignition and flame spread can be studied. Further work is needed to develop new alternative designs of PTs for the determination of AST in a variety of scenarios. It is clear that the use of plate thermometers in combination with the concept of adiabatic surface temperature will offer many opportunities to develop innovative measuring and calculation techniques in fire safety engineering.

\section{REFERENCES}

[1] Wickström, U., Duthinh, D. and McGrattan, K. Adiabatic Surface Temperature for Calculating Heat Transfer to Fires Exposed Structures, Proceedings $11^{\text {th }}$ International Interflam Conference. September 3-5, 2007, London, England, 943-953, 2007.

[2] Van de Leur, P.H.E. and Twilt, L., Fire Resistance Furnace Calibration. TNO-Report 96-CVBR1323, 1996.

[3] Wickström, U., Jansson, R. and Tuovinen, H., Experiments and theory on heat transfer and temperature analysis of fire exposed steel beams, SP Report 2009:19, ISBN 978-91-86319-03-8 (2009).

[4] McGrattan, K.B., S. Hostikka, J.E. Floyd, H.R. Baum and R.G. Rehm, Fire Dynamics Simulator (Version 5), Technical Reference Guide, NIST SP 1018-5, National Institute of Standards and Technology, Gaithersburg, Maryland, July 2005. 
[5] Wickström, U., Measurement of incident radiant heat flux with the plate thermometer, Proceedings $12^{\text {th }}$ International Interflam Conference. 5-7 July, 2010, Nottingham, England, 327-340, 2010.

[6] Holman, J.P., Heat Transfer, $4^{\text {th }}$ ed., McGraw Hill, ISBN 0-07-029598, USA, 1976.

[7] Wickström, U., 2009. Adiabatic Surface Temperature and the Plate Thermometer for Calculating Heat Transfer and Controlling Fire Resistance Furnaces. Fire Safety Science 9: 1227-1238. http://dx.doi.org/10.3801/IAFSS.FSS.9-1227

[8] Mudan, K. and Croce, P., "Fire Hazard Calculations for Large Open Hydrocarbon Fires," SFPE Handbook of Fire Protection Engineering ( $\left.2^{\text {nd }} e d.\right)$, DiNenno P.J. (ed.), National Fire Protection Association, Quincy, MA, 1995.

[9] Sterner, E. and Wickström, U., TASEF - Temperature Analysis of Structures Exposed to Fire, SP Report 1990:05, Swedish National Testing and Research Institute, Borås, 1990.

[10] Karlsson, B., Quintiere, J.G., Enclosure fire dynamics, CRC Press LLC, ISBN 0-8493-1300-7, USA, 2000.

[11] Häggkvist, A., The Plate Thermometer as a Means of Calculating Incident Heat Radiation - A Practical and Theoretical Study, Department of Civil, Mining and Environmental Engineering, Luleå University of Technology, Luleå, 2009. 